\title{
Display Technology of General Production Report on Cement Enterprise
}

\author{
Yiling Shao ${ }^{1}$, and Shaohong Jing ${ }^{2}$ \\ ${ }^{1}$ School of Electical Engineering, University of Jinan, Jinan, China \\ ${ }^{2}$ School of Electical Engineering, University of Jinan, Jinan, China
}

\begin{abstract}
Production report plays a significant role for the adjustment of industrial structure on cement enterprises. However, types of production reports are various, using general report technology can accelerate development progress and simplify report development process. This paper designs a kind of display technology of general production report based on datagrid which unifies report style, designs fixed template, realizes display function of header area and detail area on production report, and completes information management of cement enterprises
\end{abstract}

\section{Introduction}

Production reports play a vital role in the cement industry. Through the production report, cement enterprises understand the drawbacks of their own energy consumption structure, adjust the industrial structure, avoid overcapacity, and achieve energy conservation and emissions reduction. But because types of production reports are numerous, such as $\mathrm{xx}$ cement group, the main production reports are divided into two types: basic statistics and comprehensive statistics. Basic statistical reports are segmented into electricity statistics statements, basic production statistics reports, basic electric ity statistics statements, basic power consumption statistics statements and other small categories; Similarly, the consolidated statistical statements are also divided into energy-related comprehensive statistical statements, comprehensive power consumption statistical report of clin ker line, co mprehensive power consumption statistics statements of cement line and other s mall categories. Two types of production reports have more than 400 sheets in total, and the production report styles of $\mathrm{xx}$ cement group headquarters and subsidiaries are different. If taking the traditional way to create a production report for each branch separately, the workload is unpredictable. The use of general production report technology speeds up the development progress and streamlines development process of production report.

At present, there are two commonly used development methods in the common reporting system: (1) the use of third-party components available in the market, such as Crystal reports, and so on. Though its operation is simple and powerful, the price of this report system software is higher, especially for small and med ium-sized enterprises. (2) Install the report system on the server. The software need to be constantly updated and is not conducive to be put into use in bulk [1]. In order to reduce procurement costs and improve work efficiency, this paper designs a kind of display technology of general production report based on datagrid. The development platform is Visual Studio 2012, database is SQL Server 2012.

\section{Overall design}

Report is universally segmented into header area, detail area, and footer area. Header area occurs at the beginning of a report, and is usually used to display field captions and other information; Detail area is the core of the report and generally shows the actual information of the report body; Requirements on the footer for cement enterprises are not high, not too much discussion. Database stores information of header area and detail area, then the database engine executes SQL queries, through a series of processing, the browser finally shows production report, as shown in Fig. 1.

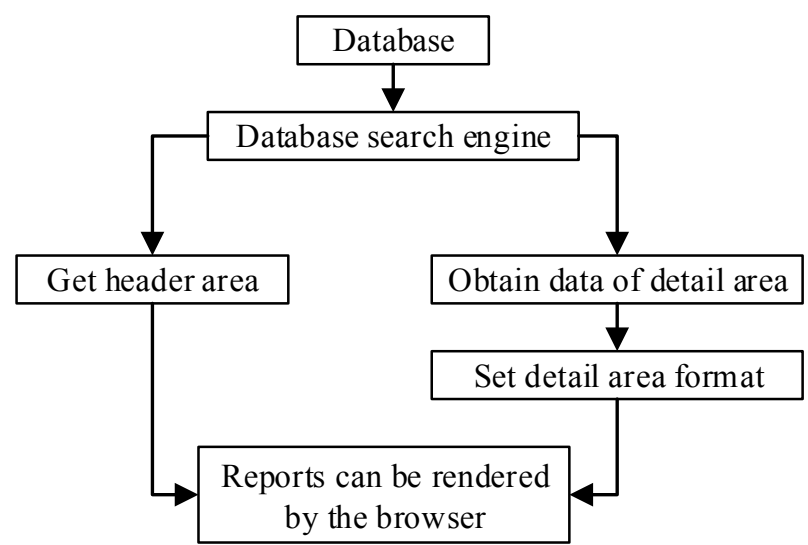

Fig. 1. Overall structural design 


\section{Related technology}

JQuery EasyUI is a collection of jQuery-based UI plugins, the goal is to help web developers more easily create a feature-rich and beautiful UI interface. Developers don't need to write complex JavaScript or have a deep understanding of CSS styles, and just know a few simple HTML tags. JQuery EasyUI provides easy-to-use components that enable Web developers to quickly build program pages on popular jQuery kernels and HTML5.

Datagrid is a set of plugins of jQuery EasyUI which extends from \$.fn.panel.defaults. It displays data in tabular format and provides rich support for selecting, sorting, grouping, and editing data. It is designed to reduce development time, and does not require the developer have specific knowledge. It is lightweight, but multifunctional which its characteristics include merg ing of cells, multi-column header, frozen columns and footers, and so on.

Datagrid has a variety of methods that can be created by using existing table elements, <table $>$ tags, or Javascript. Use Javascript to create datagrid, code is simple, therefore this article takes the way to write code.

AJAX("Asynchronous Javascript And XML") refers to a web page development technology for creating interactive web applications. By making a s mall amount of data exchange with the server in the background, AJAX can make the page asynchronously updated. This means that you can update a portion of the page without reloading the entire page. However, if the traditional web page (does not use AJAX) needs to update the content, you must reload the entire page.

$\mathrm{CH}$ is a secure, stable, simple, elegant, and objectoriented programming language which is derived fro $\mathrm{m} \mathrm{C}$ and $\mathrm{C}++$. It inherits $\mathrm{C}$ and $\mathrm{C}++$ powerful features while removing some of their complex features (such as no macro and does not allow multiple inheritance). $\mathrm{CH}$ combines simple visualization of $\mathrm{VB}$ and high operating efficiency of $\mathrm{C}++$, and is the preferred language for .NET development with powerful operating power, elegant grammar style, innovative language features and convenient component-oriented programming support.

SQL is the abbreviation for Structured Query Language. SQL is designed specifically for the database and a full-featured database language. When you use it, you only need to issue a "do what" command, "how to do" is not considered by users. SQL is powerful, easy to learn and use. It has become the basis for database operations, and now almost all of databases support SQL.

JSON (JavaScript Object Notation) is a lightweight data exchange format. JSON uses a completely language- independent text format that makes JSON become an ideal data exchange language. It is easy to read and write, but also easy to machine analysis and generation.

\section{Get header area}

The flowchart of getting header area is shown in Fig. 2.

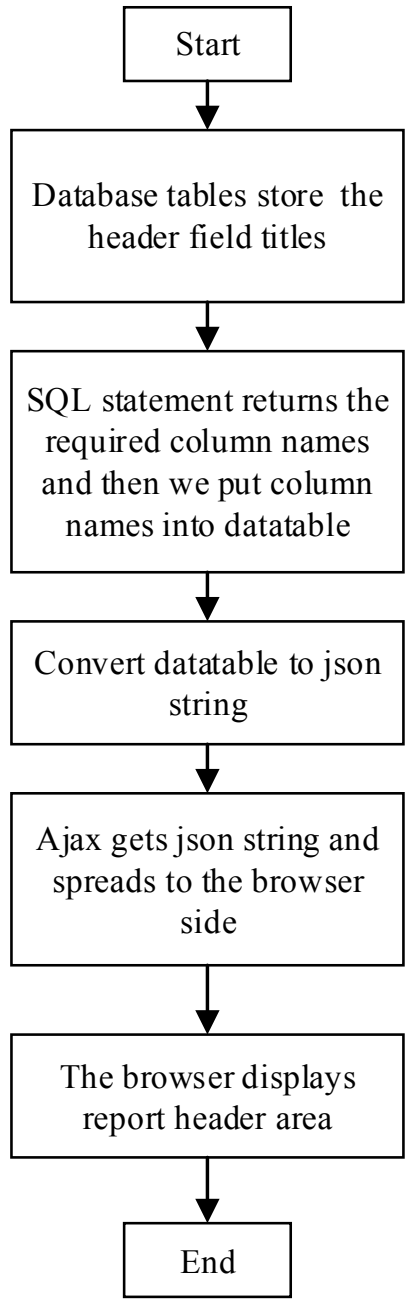

Fig. 2. The flowchart of getting header area

Specific process requires the following steps:

(1)Orig inal data of general production reports are stored in a database table and field titles of these data are mostly named after English. Such as statistics on coal consumption of $\mathrm{xx}$ cement group, create a new database table called system_TitleName, and respectively set four fields which are ID, titleName, chineseName and tableName. ID is as an identifier, and automatically generated by the system; The other three fields store English names of field titles, the corresponding Chinese names and the production report name in turn.

(2)After building the system_TitleName table, C \# connects to the database. If the connection is success ful, you need take out English names of field titles and the corresponding Chinese names from the above table and put it into datatable, conversely return error information.

(3)In order to facilitate the browser-side language Javascript's processing, you need to convert data source formats such as datatable which is queried to JSON string. When transmitting the same data, JSON is s maller than xml. First determine whether there is content in the datatable, no content to return "\" rows \": []", on the contrary, you need to follow the JSON string format to return to the contents of datatable. Data format of JSON string is generally " $\{$ "name": "Zhang three", "age": "30" \}'. JSON holds objects with curly braces which can contain multiple name / value pairs. 
(4)AJAX requests JSON data and Javascript parses with it. AJAX have type, url, contentType, dataType, success, and so on several common parameters. If the request succeeds, the JSON data are invoked in the success method. This method has two parameters: the data returned by the server, and the state returned. The format of the call is as follows:

function (data, textStatus)

// data could be xmlDoc, JSONObj, html, text, etc...

this; // the options for this AJAX request \}

The key code is as follows:

success: function $(\mathrm{msg})$ \{

var $\mathrm{m}$ msg = JSON.parse(msg.d);

//Convert the JSON string into a Javascript object

if $(\mathrm{m}$ ms g.total $=0)\{$

alert("error!");

\}

else

\{

var titnames $=[]$;

for (var $\mathrm{i}=0 ; \mathrm{i}<\mathrm{m}$ msg.rows.length; $\mathrm{i}++)\{$

titnames.push( $\left\{\right.$ filed: $m \_m s g . r o w s[i] . t i t l e N a m e$, title: $m \_m s g . r o w s[i] . c h i n e s e N a m e$, width:100\});

\}

\$("\#Windows_Report").datagrid( \{

striped: true,

rownumbers: true,

singleSelect: true,

fit: true,

pagination: true,

pageSize: 50 ,

toolbar:'\#toolbar_ReportTemplate',

columns: [titnames]

\}$)$

\}

Datagrid columns are the column configuration objects of the data grid (datagrid) whose properties are objects. To dynamically add the columns property, you must assign values strictly in accordance with its attributes format. Create an array called titnames, push titleName, chineseName fields into it one by one according to the attributes of columns, and assign values to the datagrid columns. The field titles in a web page can be displayed in Chinese correctly and easily.

\section{Detail area's content acquisition and format settings}

\subsection{Detail area obtains production data}

Details' data are the core part of the cement production report which can represent the current production levels of the cement enterprises in some extent and provide objective basis for the enterprise production target in the future.

Obtaining the content of the detail area is similar to that of the header field, specific process is as follows:
1)Connect to the database

2)SQL statement query and return the report production data

3)Put the queried report production data into datatable

4) Transfer datatable into JSON string

5)AJAX gets and deals with JSON string

6) The web page shows the production data of detail area

\subsection{Detail area sets format}

Types of production data are roughly divided into two categories, the numerical and the non-numerical. Determines the data type, the numeric is right justified, the non-numerical is left-aligned.

Many of production data content are more. Setting the width adaptively is easy to appear scroll bars which are likely to affect the appearance of the web page. Set the fixed width, if the production data exceed this width, then the excess will be displayed in the form of ellipses; When the mouse touches the excess, the browser will show the entire production data.

Use the datagrid's formatter property to format the detail. The core code is as follows :

function formatCellTooltip(value) \{

var reg $=$ new $\operatorname{Reg} \operatorname{Exp}(" \wedge[-+] ?[0-9]+(\backslash .[0-9]+) ? \$ ")$;

if (reg.test(value))

return '<span style="float: right " $>$ ' + value + '</span $>$;

return $"<$ span title="'+ value + "'>" + value

$+"</$ span $>$;

\}

\section{Conclusion}

This paper presents a kind of general display design idea for the production report of cement enterprises. This paper, by using database to store the data of the table names and subsidiary area in the production report, and then through the SQL statements, c\# datatable, JSON and Javascript and so on a series of processing, eventually makes production reports have common display function. How do I make the header area with multilayer structure, detail area's format more variety as well as display functions of general production report more efficient, which is the next step to be solved.

\section{Acknowledgment}

This work is supported by The Research Fund for the Taishan Scholar Project of Shandong Province of China, Independent Innovation and Achievements Conversion of Shandong Province under Grant 2015ZDXX0101F01 and Independent Innovation and Achievements 
Conversion of Shandong Province under Grant 2014 CGZH0601.

\section{References}

1. Baidong JIN. Design of Universal Report Based on Self-defined Template. Journal of computer age, 2009; $10: 38-39$.

2. Xiaojuan Zhou. Design of General Report System in Web. Railway Computer Application. 2004; 13(5) :43-44.

3. Weihu Kang. Excel reporting design and implementation based on the. NET. Soft ware Guide, 2016; 15(5):101-103.

4. Fengxia Cong. Research on Automatic Generation Technology of General Crystal Report. Computer technology and development, 2013;23(5): 54-57+62

5. Xiaochun Yu. General Report Programming. Microcomputer Applications, 2000; 16(10): 61-54 